\title{
„Das Patientenrechtegesetz bestätigt den Heilpraktiker als Heilberufu
}

Seit 2013 ist verständlich in einem Gesetz geregelt, was Patienten von Behandelnden erwarten dürfen. Diese Klarheit haben viele begrüßt, insbesondere Patientenverbände. Kritiker sahen darin eine Gefahr für die Zunahme von Prozessen wegen Behandlungsfehlervorwürfen. Siegfried Kämper hat als Leiter der Gutachtenkommission des BDH aufmerksam verfolgt, welche Auswirkungen das Patientenrechtegesetz für Heilpraktiker hat. Sein Fazit: Kaum Nachteile.

Herr Kämper, das neue Patientenrechtegesetz: Gut oder schlecht für Heilpraktiker?

Gut. Meiner Meinung nach hat das Gesetz für uns kaum Nachteile. Es enthält weder Schikanen noch beschränkt es uns in unserer Arbeit. Im Gegenteil, es hat für uns Vorteile: Es bestätigt den Heilpraktiker als Heilberuf. Im Gesetzestext ist nicht vom „Arzt“, sondern stets vom „Behandelnden“ die Rede. Dadurch wird deutlich, dass es neben dem Arzt auch andere Heilberufe gibt, und neben den Ärzten haben wir Heilpraktiker die größte Behandlungsautonomie. Das Patientenrechtegesetz fasst zudem nicht nur die Rechte der Patienten zusammen, sondern auch explizit die Pflichten der Behandelnden.

Und das ist ein Vorteil.

Klar, in dieser Deutlichkeit war bisher noch nirgends nachzulesen, welche Verpflichtungen bestehen bezüglich der Behandlung, und vor allem, welche gegenüber den Patienten. Bisher galt, was zum Dienstvertrag im BGB steht. Dank der Konkretisierung im Patientenrechtegesetz hat nun jeder die Chance, in seiner Praxis alles richtig zu machen. Und das sehe ich als einen weiteren Vorteil: Dank dieser Transparenz ist das Bewusstsein bei den Behandelnden und den Patienten sowohl für die Rechte als auch die Pflichten gewachsen.

Ein Behandlungsvertrag, und darum geht es für uns im Patientenrechtegesetz ja im Wesentlichen, ist eine Vereinbarung zwischen Patient und Behandelndem. Gerade das Arbeiten an und mit leidenden Menschen ist sehr individuell zu gestalten, und deshalb ist unser Behandlungsvertrag

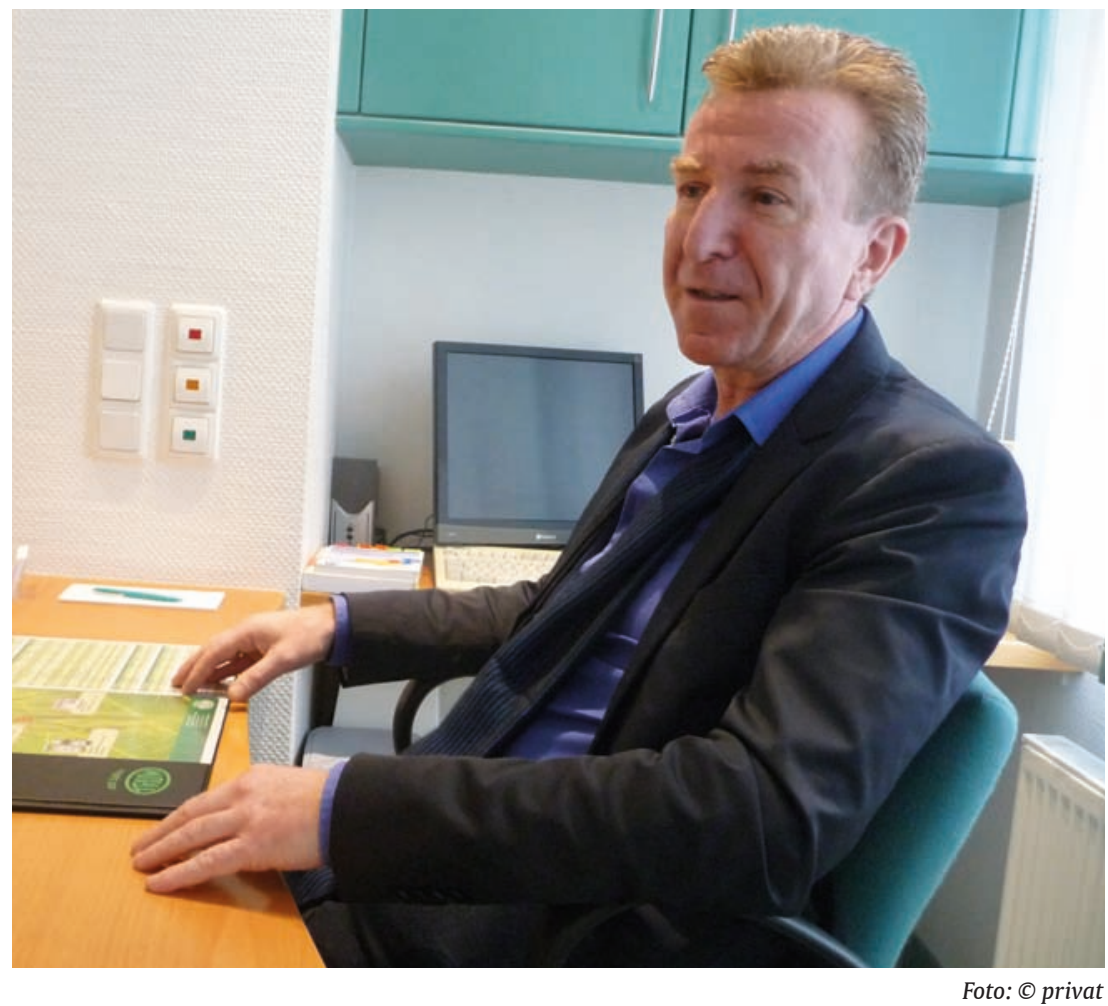

nicht mit dem Dienstvertrag z. B. eines Handwerkers gleichzusetzen, bei dem es um eine Reparatur geht. Jeder, Patient und Behandelnder, kann jetzt nachlesen, worauf es in diesem Behandlungsvertrag ankommt - aber nicht nur in der Absprache, der Aufklärung und der Dokumentation, sondern auch in der Ausführung einer Behandlung. Denn das Patientenrechtegesetz fordert auch, dass eine Behandlung stets nach dem gültigen allgemeinen Standard zu erfolgen hat. Diesen mussten wir Heilpraktiker natürlich bisher auch einhalten. Aber so deutlich stand das zuvor in keinem Gesetz. Bisher musste man das indirekt aus vielen unterschiedlichen Regelwerken, beispielsweise der TRBA 250 oder den RKI-Richtlinien, herauslesen und entsprechend interpretieren.

Gibt es Fallstricke im Patientenrechtegesetz?

Kaum, und das sehe ich wirklich als wesentlichen Vorteil des Patientenrechtegesetzes. Es gibt nur einen Punkt, der leider ein Fallstrick werden kann. Auf den weise ich Kollegen deshalb in meinen Vorträgen immer besonders hin: Wenn eine vollständige Kostenübernahme durch die Beihilfe oder den privaten Krankenversiche- 
rer nicht gesichert ist, verlangt das Patientenrechtegesetz, dass der Heilpraktiker dies seinem Patienten vor der Behandlung schriftlich mitteilt. Dazu müsste der Patient allerdings selbst wissen, und das seinem Heilpraktiker dann auch sagen können, für welche Leistungen und in welchem Umfang er versichert ist. Kaum ein Patient weiß das jedoch genau. Und die wenigsten Patienten wissen zudem, ob ihr Versicherer die anfallenden Kosten für die sogenannten wissenschaftlich nicht anerkannten Verfahren überhaupt erstattet bzw. in welcher Höhe, und ob eine Selbstbeteiligung im Vertrag festgelegt ist. Es gibt hier gerade bei den Zusatzversicherungen etliche unterschiedliche Modelle. Der Behandelnde müsste also den Patienten bitten, seinen Vertrag mit in die Praxis zu bringen, er müsste diesen gemeinsam mit ihm durchgehen und dann seinem Patienten erläutern, welche Behandlungen der Versicherer erstattet und welche wahrscheinlich bzw. sicherlich nicht. Dazu muss der Heilpraktiker natürlich wissen, welches die nicht wissenschaftlich anerkannten Verfahren sind. Er muss zudem nachfragen, was der Versicherer evtl. in der Vergangenheit schon zu erstatten abgelehnt hat, wie hoch vielleicht früher bei vergleichbaren Behandlungen die Selbstbeteiligung war etc. All das kann man leider fordern von einem Heilpraktiker, er ist ja Profi. Der Gesetzgeber sieht den Heilpraktiker eben auch als einen Dienstleis- ter, vergleichbar mit einem Handwerker, der natürlich auch ungefähr den Kostenaufwand abschätzen können muss, der auf seinen Kunden zukommt. Aber es ist ein enormer Mehraufwand, zu dem der Gesetzgeber uns Heilpraktiker hier verpflichtet, und den können wir in diesem Umfang nicht immer leisten. Deshalb habe ich für die Abrechnungsfibel des BDH eine Honorarvereinbarung formuliert, die es dem Behandelnden ermöglicht, die geforderte Textform zu liefern, sofern er sich nicht imstande sieht, dem Patienten vorab mitzuteilen, wie hoch dessen mögliche Selbstbeteiligung an der Behandlung sein wird (s. Musterformular S. 56).

\section{Wirkt sich das Patientenrechtegesetz} anderweitig spürbar in der Praxis aus? Die vielfältige Berichterstattung in den Medien hat Patienten auf ihre Rechte aufmerksam gemacht, und die nehmen sie nun auch verstärkt für sich in Anspruch. Insbesondere das Recht auf Einsichtnahme in die Patientenakte fordern viele für sich ein. Ich bekomme etwa zwei E-Mails im Monat mit der Anfrage von einer Kollegin bzw. einem Kollegen, wie sie sich verhalten sollen, weil ein Patient oder ein Versicherer die Patientenakte zur Ansicht angefordert hat, und zwar unverzüglich. Früher kamen solche Anfragen vielleicht einmal in zwei Jahren und dann waren es fast ausnahmslos Einsichtnahmen wegen Behandlungsfehlervorwürfen.
Warum werden die Akten heute angefordert?

Meist geht es darum, dass ein Versicherer eine Leistung nicht erstatten will oder eine Behandlung infrage stellt. Es kommt aber auch vor, dass der Patient den Heilpraktiker wechseln will, obwohl er mit der Behandlung bisher zufrieden war, und genauso weiterbehandelt werden möchte wie bisher. Meist spielt Geld dabei eine Rolle. Der Patient denkt, dass sich die erfolgreiche Behandlung eins zu eins aus der Akte ableiten lässt.

\section{Was raten Sie dann?}

Niemals die Akte im Original herausgeben, sondern stets nur eine Kopie. Alle Eintragungen in der Kopie schwärzen, die persönlich oder vertraulich sind. Die müssen weder dem Patienten noch dem Versicherer gezeigt werden. Wenn ein Versicherer die Patientenakte anfordert, die Einverständniserklärung des Patienten vorlegen lassen, niemals die Akte ohne diese herausgeben. Zudem rate ich, wenn ein Versicherer die Akte einsehen will, in Absprache mit dem Patienten zunächst nur einen Befund- und Behandlungsbericht zu schicken, meist begnügen sich die Versicherer damit. Der Befund- und Behandlungsbericht muss aber zuvor dem Patienten vorgelegt werden und der muss ihn absegnen.

Sie sagten es ja schon, manchmal wird die Akte auch wegen eines Behand- 
lungsfehlervorwurfs angefordert. Ist das Patientenrechtegesetz in Sachen Behandlungsfehlervorwurf für den Heilpraktiker eher von Vorteil oder Nachteil?

Es hat sich im Grunde ja nichts geändert. Allerdings sind die Patientenrechte jetzt klar in einem Gesetz festgeschrieben, und das ermutigt eben manche Patienten geradezu, schon beim geringsten Verdacht auf einen Behandlungsfehler aktiv zu werden. Für den Heilpraktiker bedeutet das dann eben einen unbezahlten Mehraufwand, wenn die Patientenakte angefordert wird. Darin kann man schon einen Nachteil sehen. Wie ich das sehe, ist die Zahl der wegen Behandlungsfehlervor- würfen geführten Verfahren aber nicht gestiegen.

\section{Was raten Sie Kollegen?}

Ich empfehle für alle, insbesondere aber für die invasiven Verfahren wie Injektions- und Infusionstherapien, Ozon-Eigenblutbehandlung etc., aber auch für die Chiropraktik oder Osteopathie, Checklisten $\mathrm{zu}$ erstellen, die die Arbeitsweise Schritt für Schritt beschreiben. So etwas nennt man Arbeitsausweis. Somit ist für jede Behandlung das Vorgehen nachvollziehbar dokumentiert und kann im Bedarfsfall beurteilt werden. Ganz wichtig in diesem Zusammenhang ist der Hygieneplan, der ebenfalls in keiner Praxis feh- len sollte. Und der sollte natürlich dem aktuellen Stand entsprechen und regelmäßig aktualisiert werden.

Ein einrichtungsinternes Qualitätssicherungsmanagement, eine gute Dokumentation der Arbeitsschritte und der Praxisführung und natürlich eine gute Dokumentation in der Patientenakte, die den Vorgaben des Patientenrechtegesetzes entspricht: Wer sich daran hält, ist im Falle eines Behandlungsfehlervorwurfs gut gerüstet. "

\section{Herr Kämper, vielen Dank für das Gespräch.}

Dieser Artikel ist online zu finden: http://dx.doi.org/10.1055/s-0035-1549145

\section{Honorarvereinbarung/Aufklärung über möglichen Selbstbehalt bei Erstattungs- anspruch durch einen Kostenträger (Beihilfe und/oder Privatkrankenkasse)}

Meine Honorare berechne ich gemäß Gebührenverzeichnis für Heilpraktiker (GebüH), herausgegeben von den Heilpraktikerverbänden 1985, Neuauflage 1.1.2002. Die Leistungen, die im GebüH gelistet sind, werden von mir wie folgt berechnet: Gemäß Erstattungstabelle (Anlage zur Honorarvereinbarung) Spalte

Ich möchte Sie darauf aufmerksam machen, dass viele private Krankenkassen unterschiedliche Tarife anbieten, sodass je nach Behandlung manchmal nur der GebüH-Mindestbetrag, manchmal aber auch bis zum Höchstbetrag erstattet wird. Auch die Beihilfe und Postbeamtenkrankenkasse erstattet nicht jeden Betrag und i. d. R. nur bis zur Höhe des in der Erstattungstabelle Spalte genannten.

Obschon die im GebüH gelisteten Beträge älter sind als ein viertel Jahrhundert, und somit nicht mehr zeitgemäß, berechne ich bei beihilfeberechtigten Patienten (Beamte) generell nur die Beträge, die das Bundesinnenministerium erstattet (= Spalte ). Sollte Ihre Zusatzversicherung oder Privatkrankenkasse abweichend erstatten, was aus Ihren mir nicht vorliegenden Vertragsunterlagen zu entnehmen ist, kann es zu einer zumutbaren Selbstbeteiligung kommen. Das Patientenrechtegesetz verpflichtet mich, Sie darüber aufzuklären, dass möglicherweise nicht der gesamte Betrag und jede erbrachte Leistung erstattet werden, die ich Ihnen in Rechnung stelle.

Leistungen mit fehlendem Betrag in der entsprechenden Spalte sind nicht beihilfefähig.

Nicht alle inzwischen möglichen und etablierten Verfahren, die ich anbiete, sind im GebüH und der Erstattungstabelle aufgeführt. Analoge Abrechnung bedeutet, dass ich dann eine (oder mehrere) Leistung(en) auf der Rechnung benennen kann, die der erbrachten Leistung am ähnlichsten ist/sind. Die Erstattung analog abgerechneter Leistungen wird von den Kostenträgern unterschiedlich gehandhabt. Erstattungssicherheit besteht nicht.

Folgende Leistungen sind im GebüH nicht gelistet oder werden von mir abweichend wie folgt berechnet:

Bitte prüfen Sie Ihren Versicherungsvertrag (ggf. durch persönliche Nachfrage bei der Versicherungsgesellschaft) dahingehend, ob die vorgesehene Behandlung (vorgesehene[s] Therapieverfahren)

anerkannt ist und wenn ja, in welcher Höhe die Leistung erstattet wird.

Wenn Sie trotz dieser Auskunft noch Fragen haben oder noch nicht sicher wissen, ob eine Zuzahlung auf Sie zukommt bzw. in welcher Höhe, können Sie mir die mit Ihrem Kostenträger vereinbarte Leistungszusage vorlegen. Wir können dann gemeinsam die mögliche Selbstbeteiligung ausrechnen.

Mit der Unterschrift bestätigen Sie, dass ich Sie ausreichend über meine Abrechnungspraxis und eine mögliche Selbstbeteiligung aufgeklärt habe.

Ort, Datum der Aufklärung

Unterschrift des Patienten

Text aus: @ BDH-Fibel; 2015 (modifiziert) 